\title{
Diagnostic Utility of AMCAR and p63 Cocktail Antibody in the Benign and Malignant Lesions of Prostate
}

\author{
Sujitha Chougani ${ }^{1}$, Sunandalakshmi* GV$^{2}$, Durga Kharidehal ${ }^{2}$, Ravi Sankar $V^{3}$ and Santhi Vissa ${ }^{2}$ \\ ${ }^{1}$ Yashoda Hospital, Secunderabad, Telangana \\ ${ }^{2}$ Department of Pathology, Narayana Medical College, Nellore, Andhra Pradesh \\ ${ }^{3}$ Department of Emergency Medicine, Narayana Medical College, Nellore, Andhra Pradesh.
}

\begin{abstract}
Backgorund: Histopathological examination of prostatic specimen is gold standard for the diagnosis of prostate cancer. Current study evaluates the expression of AMACR and p63 in the prostate lesions using AMACR and p63 cocktail.

Materials and Method: Total of 180 cases were collected and Haematoxylin and Eosin staining performed followed by immunohistochemical analysis using AMACR and p63 antibody.

Result: Out of 180 cases, Benign Prostatic Hyperplasia is the most common lesion noted in about 120 cases. In this study, the predominant population was in the 6 th to 7 th decade of age. Most of the patients presented with difficulty in micturition. Immunohistochemistry revealed that p63 expression is positive in all normal basal cells, 118 cases $(98.33 \%)$ were negative for AMACR and only 2 cases were showing focal and weak AMACR immunoreactivity. AMACR was positive in all the 6 HGPIN cases with variable intensity. Out of 5 LGPIN cases AMACR was positive in 3 cases with low intensity, remaining 2 cases shows AMACR negative. p63 is positive in all 11 PIN cases (LGPIN \& HGPIN) showing discontinuous staining pattern. All 22 cases of Prostatic adenocarcinomas were negative for p63 and all cases expressed positive immunostaining with AMACR. A diagnosis of adenocarcinoma was made in $48 \%$ of atypical cases. Cases which were negative for both AMACR and p63 were diagnosed as Atypical Small Acinar Proliferation, for which further follow-up is required.
\end{abstract}

Conclusion: AMACR/p63 Cocktail antibody is very much useful as it saves time, tissue and is cost-effective.

Keywords: Immunohistochemical Analysis, Prostate Lesions, AMACR/p63 Cocktail Antibody.

\section{Introduction}

Benign prostatic hyperplasia is a common urological condition in men. The prevalence of BPH increases from $20 \%$ at 40 years of age to $90 \%$ by the eighth decade of life. ${ }^{[1]}$ Prostate cancer is globally the second frequently diagnosed cancer and the sixth leading cause of cancer death in males. ${ }^{[2]}$ In India, it constitutes about $5 \%$ of all male cancers. ${ }^{[3]}$

Prostate specific antigen (PSA), digital rectal examination and transrectal ultrasound are the tools most commonly used to screen for prostate cancer. However, histopathological examination remains the gold standard for final diagnosis. Histological diagnosis of prostate cancer is usually based on morphological features such as growth pattern, nuclear atypia, and absence of basal cells.

However, this can be challenging particularly when the malignant tissue is limited and is mixed with benign prostate glands, or because of benign mimickers of carcinoma ${ }^{[4]}$ Most mimics of prostatic carcinoma fit within the small gland category. In such a case, the diagnosis of atypical small acinar proliferation, suspicious but not diagnostic of prostatic carcinoma is made. The application of immunohistochemistry may be essential to confirm the diagnosis and/or distinguish prostatic carcinoma from the benign mimics.

One feature distinguishing cancer mimics from prostate cancer is that benign glands contain basal cells, which are absent in cancerous glands. pathologists often use immunohistochemical markers to label basal cells when faced with an ambiguous lesion. ${ }^{[5]}$ The most commonly used marker is high molecular weight cytokeratin (34bE12) and more recently, markers such as p63, CK5, CK5/6 and CK 14 have been proposed. ${ }^{[6[17][8][9][10]}$ AMACR is used as cancer associated positive marker. ${ }^{[1]}$ In the workup of difficult cases, AMACR has been proposed as a positive prostate cancer marker in conjunction with negative basal cell markers (p63 and high molecular weight cytokeratins).

The accurate pathological evaluation of prostate lesion is essential, because the subject of prostatic disease is fraught with doubts, uncertainties, and apparent contraindications.

This study was undertaken to study the role of AMACR and p63 cocktail in different benign and malignant lesions of the prostate, especially in suspicious or atypical cases whenever possible. 
Present study aimed to study the utility of AMACR and p63 cocktail in suspicious cases in reaching a definitive diagnosis.

\section{Materials and Methods}

The present study is a prospective study. Study is conducted for a period of two years from August 2013 to July 2015.

The study is based on evaluation of AMACR and p63 expression in prostatic lesions observed in 180 prostatic specimens received at the Upgraded Department of Pathology, Osmania General Hospital, Afzalgunj, Hyderabad.

\section{Inclusion Criteria:}

- This study includes transurethral resected prostatic specimens, prostate needle biopsies, Open prostatectomies collected over a period of 2 years.

- Due importance was given to record a brief clinical history with age, Inpatient registration number, biopsy number, serum PSA levels.

The specimens were received in $10 \%$ formalin. After gross examination, sections were processed for one day and later embedded in paraffin which was cut at fivemicron thickness. Sections were stained with conventional Haematoxylin and Eosin stains.

A total of 180 cases are included in the study. Out of 180 cases, 129 TURP specimens, 47 needle biopsies and 02 open prostatectomy specimens. The H and E stained slides of all the cases were reviewed and a provisional diagnosis was made. All these 180 cases were subjected to immunohistochemical marker AMACR and p63 cocktail.

Hematoxylin and eosin (H\&E) performed as standard procedure.

Immunohistochemistry performed as standard procedure using antibody cocktail.

Primary antibody: Anti-AMACR+ p63 (4A4), prediluted (Abcam product, catalog no -ab82747).

Slide evaluation was performed by using a Light microscope. AMACR stains a variety of normal and neoplastic tissues. Positive AMACR staining is uniformly described as being easily visible on low power examination, as circumferential, granular, luminal (apical) to subluminal and diffusely cytoplasmic in nature.

Observed AMACR staining showed following grades of staining intensity in accordance with Luo $\mathrm{J}$ and $\mathrm{Zha} \mathrm{S}$ et al. ${ }^{[12]}$

\section{Grade staining pattern}

0 When there is absolutely no staining observed.

$1-10 \%$ cells in a gland show Positive staining.
$10-50 \%$ cells in a gland show Positive staining.

$>50 \%$ cells in a gland show Positive staining.

\section{Morphological evaluation of p63}

Immunohistochemical expression was assessed semiquantitatively for staining intensity and percentage of positive tumor cells with brown nuclear staining. Only moderate or strong staining in at least $5 \%$ of the tumor cells was considered positive.

The whole section was scanned at low power in order to assess the general level of intensity throughout. The average intensity of the staining corresponds to the presence of negative, weak, moderate, and strong staining.

\section{Results}

Out of 180 cases, there were Benign prostatic hyperplasia120 (67\%), Prostatic intraepithelial neoplasia-11 (06\%), Suspicious cases- 27 (15\%), Prostatic adenocarcinnoma$22(12 \%)$.

The age of the patients included in this study were ranged between 46 to 83 years.

\section{Clinical features:}

Patients included in this study presented with combinations of symptoms i.e., $85 \%$ of patients presented with Difficulty in micturition $(85 \%)$, frequency $(5 \%)$, and pain $(3 \%)$. The most common symptom observed in both benign prostatic hyperplasia and prostatic adenocarcinoma patients is difficulty in micturition.

\section{Benign prostatic hyperplasia:}

The results in the present study indicate that out of 120 benign prostatic hyperplasias, 118 cases showed negative or focal, weak and non- circumferential brown cytoplasmic positivity with AMACR in the luminal cells of glands (Figure 1)

Only 2 out of 120 benign prostatic hyperplasias showed positive AMACR immunoreactivity, which is circumferential to non-circumferential luminal positivity of grade 1 intensity (Figure 2, \& Figure 3).

In all the 120 cases, p63 showed brown nuclear positivity in the basal cells of glands.

\section{Prostatic Intraepithelial Neoplasia:}

AMACR positivity was observed in 9 out of 11 PIN (HGPIN-6; LGPIN-5) cases. They showed variable intensity ranging from grade 1 to grade 3 .

The two cases were negative for AMACR expression belongs to LGPIN.

p63 was positive in 11 cases of PIN in the basal cells of glands in a discontinuous fashion. 


\section{Prostatic Adenocarcinoma:}

Out of 22 prostate adenocarcinoma cases, 22 cases showed strong AMACR expression.

In our study we observed that the staining intensity decreased as the tumor differentiation progressed from well differentiated to poorer differentiation(Figure $4, \&$ Figure 5).

\section{Suspicious Cases:}

Out of 27 cases with atypical foci, foci which were showing positive immunostaining for p63 and negative immunostaining for AMACR were considered as benign, lesions that were positive for AMACR and negative for p63 were considered as adenocarcinomas, lesions which were negative for both AMACR and p63 were diagnosed as Atypical small acinar proliferation .

Table 1: Age distribution of patient

\begin{tabular}{|l|l|l|l|l|}
\hline AGE & BENIGN & $\begin{array}{l}\text { PROSTATIC } \\
\text { INTRAEPITHELIAL } \\
\text { NEOPLASIA(PIN) }\end{array}$ & MALIGNANT & ATYPICAL LESIONS \\
\hline$<50$ & 05 & 02 & 01 & 03 \\
\hline $51-60$ & 19 & 03 & 03 & 09 \\
\hline $61-70$ & 53 & 04 & 07 & 08 \\
\hline $71-80$ & 36 & 03 & 09 & 05 \\
\hline $81-90$ & 04 & 01 & 02 & 02 \\
\hline TOTAL & $\mathbf{1 2 0}$ & $\mathbf{1 1}$ & $\mathbf{2 7}$ & \\
\hline
\end{tabular}

Table 2: Serum PSA levels in all the cases.

\begin{tabular}{|l|l|l|}
\hline & Range(ng/ml) & Mean \pm SD \\
\hline Benign & $0.8-14.4$ & $3.7 \pm 1.1$ \\
\hline PIN & $1.3-7.2$ & $4.8 \pm 1.4$ \\
\hline Atypical cases & $1.7-21.6$ & $6.8 \pm 2.3$ \\
\hline Malignant & $3.3-61$ & $19.2 \pm 4.7$ \\
\hline
\end{tabular}

Table 3: Expression of AMACR and p63 in atypical foci.

\begin{tabular}{|l|l|l|l|}
\hline & $\begin{array}{l}\text { AMACR- } \\
\text { p63+ }\end{array}$ & $\begin{array}{l}\text { AMACR + } \\
\text { p63- }\end{array}$ & $\begin{array}{l}\text { AMACR- } \\
\text { p63- }\end{array}$ \\
\hline $\begin{array}{l}\text { TURP specimens } \\
\text { (17 cases) } \\
\begin{array}{l}\text { Needle biopsies } \\
\text { (10 cases })\end{array}\end{array}$ & 08 & 06 & 03 \\
\hline Total & 02 & 07 & 01 \\
\hline
\end{tabular}

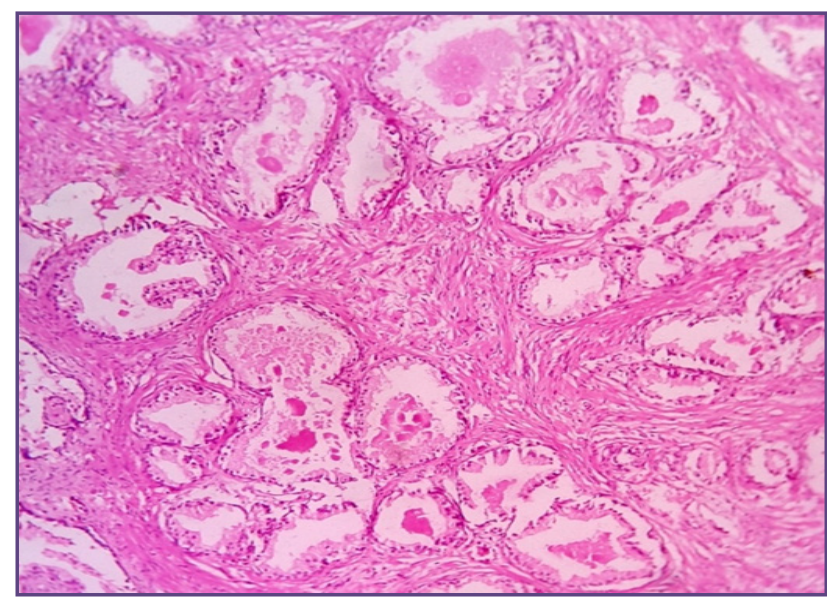

Fig. 1: Section showing both glands and stroma (benign prostatic hyperplasia). Medium to large glands with 2 benign cell layers (secretory and basal) are seen (10x)

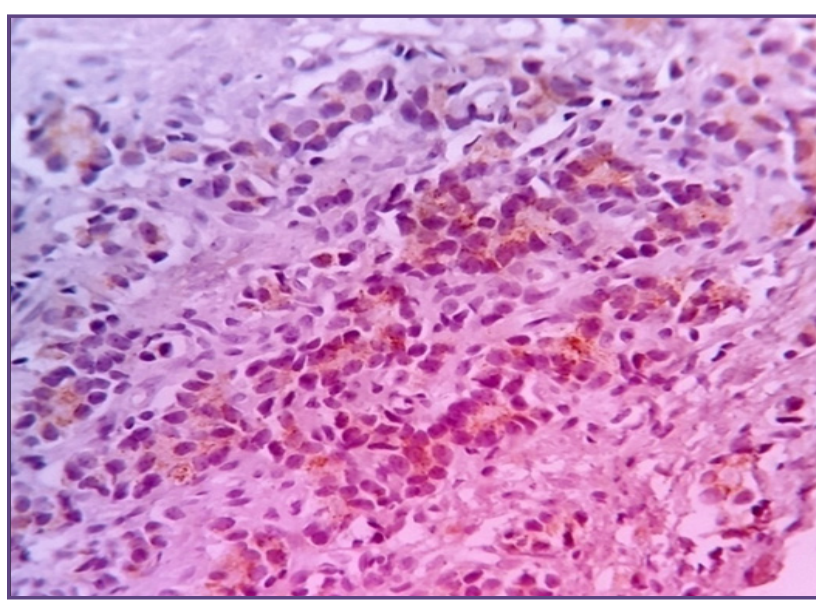

Fig. 2: Immunohistochemistry, AMACR is showing diffuse cytoplasmic positivity in tumor cells (40X). 


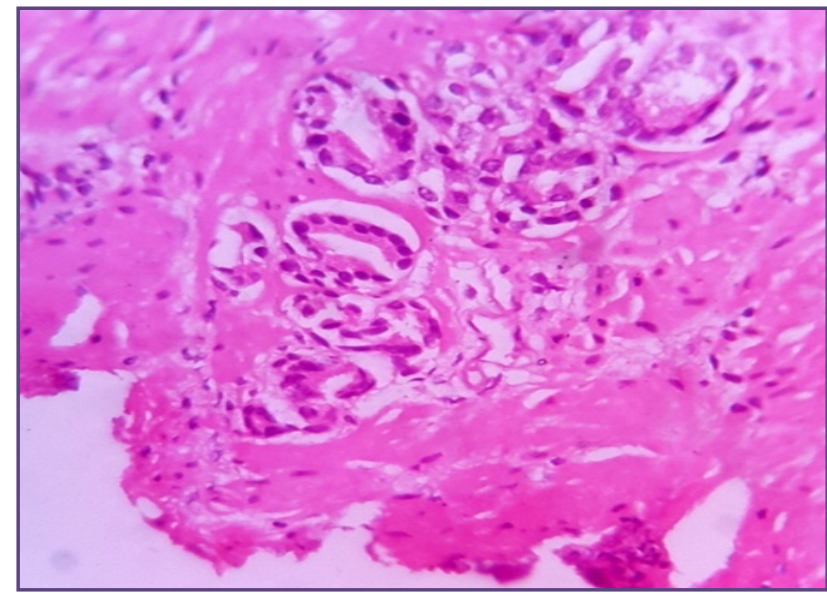

Fig. 3: Section showing atypical foci, composed of few small crowded glands lined by single layer of cells. (Malignant) H \& E (40X).

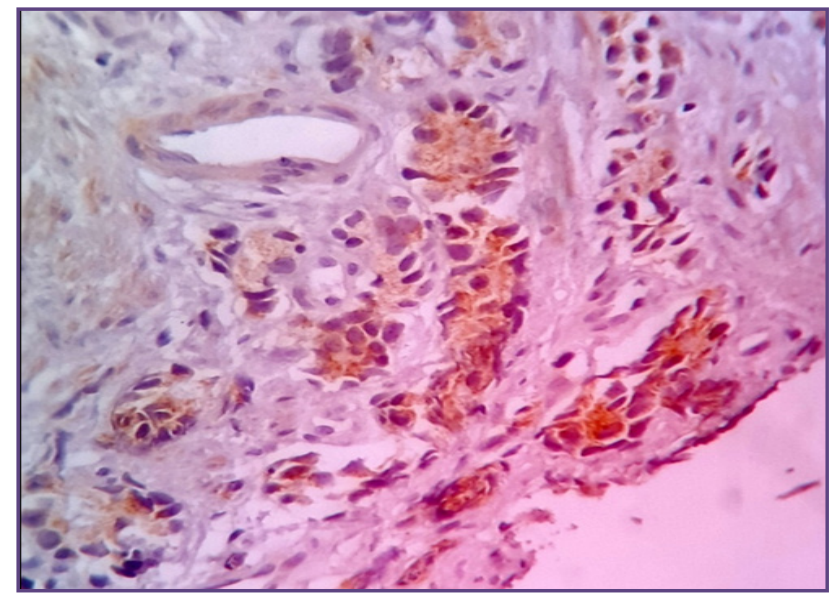

Fig. 4: IHC, AMACR is showing diffuse cytoplasmic positivity in tumor cells $(40 \mathrm{X})$. This case diagnosed as adenocarcinoma.

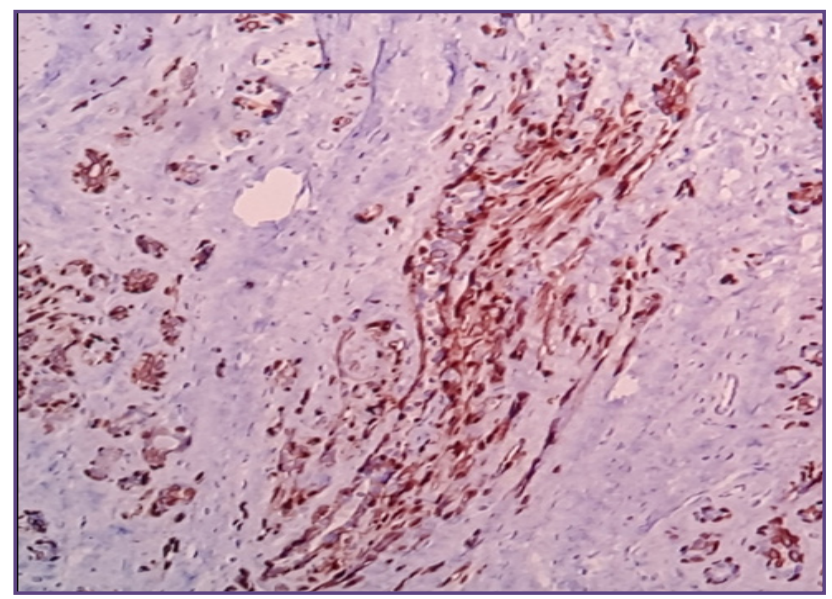

Fig. 5: Immunohistochemistry, p63 is showing nuclear positivity in the basal cells. (10 X).

\section{Discussion}

Histopathological examination of prostatic specimen is gold standard for the diagnosis of prostate cancer. The diagnosis of prostate cancer based on morphological features, frequently challenging due to the presence of either a small focus of cancer or due to the presence of many benign mimickers of malignancy.

In recent years, IHC using antibodies against basal cell markers like p63, HMWCK and CK5/6 and prostatic adenocarcinoma specific biomarker AMACR have been used as adjuvant to morphology in diagnostically challenging cases.

Placing the antibodies into a single cocktail does not alter the antigenic properties of the individual antibodies. The simultaneous application of 2 antibodies against 2 distinct, compartmentally localized proteins in prostatic basal cell nuclei and the cytoplasm of malignant prostatic epithelial cells would provide for efficient use of limited tissue in the diagnosis of adenocarcinoma in biopsy specimens especially in core needle biopsy specimens.

This study is compared with other different studies having certain similar parameters in comparison. The findings in the present study are as follows:

The age of patients in our study ranged from 46-83 yrs; however, the predominant population was in 6th to $7^{\text {th }}$ decade with a mean age of 65.6 yrs. No significant difference was noted in the mean age of the non-neoplastic and neoplastic groups. The results of the present study agree with the studies by George and Thomas et al. ${ }^{[13]}$, Barakzai et al. ${ }^{[14]}$. The decline in the number of cases beyond the age of 80 years reflects the average life span of people in our country.

In our study, benign prostatic hyperplasia was the most common lesion and accounting for about $67.7 \%$. This agrees with the studies conducted by Anjorin et al. ${ }^{[15]}$ Our results revealed high expression of p63 in 100\% of normal basal cells. Only one case showed patchy staining of p63 in few glands. In our study, out of $120 \mathrm{BPH}$ cases, 118 cases (98.33\%) were negative for AMACR and only 2 cases were showing focal and weak AMACR immunoreactivity.

According to Evans et al., pseudoneoplasms (atypical adenomatous hyperplasia, atrophy, post atrophic hyperplasia, basal cell metaplasia) shows AMACR reactivity. ${ }^{[16]}$

We reassessed the two positive cases and ruled out Pseudoneoplasms. According to Leav et al. and 
Ananthanarayana et al. a phenomenon called "Field effect" plays a role in such AMACR positive BPH cases. ${ }^{[17][18]}$

Field effect is a phenomenon in which if there is adjacent carcinoma foci, even the benign glands express the AMACR immunostaining. This is due to upregulation of metabolic pathways of prostate glands during the carcinogenic process.

In both the cases, p63 staining pattern was observed. One of the two cases showed strong basal cell nuclear positivity in all the glands explaining the phenomenon of over staining. The other case was found to be showing patchy staining in some of the glands and supported the phenomenon of Field effect and advised the urologist for multi core biopsy, but unable to follow up the patient.

In our study AMACR was positive in all the $6 \mathrm{HGPIN}$ cases with variable intensity. Out of 5 LGPIN cases, AMACR was positive in 3 cases with low intensity; in the remaining 2 cases AMACR was negative. p63 is positive in all the 11 PIN cases (LGPIN \& HGPIN) showing a discontinuous staining pattern.

In Sanderson et al. study ${ }^{[19]}$ all the HGPIN cases showed p63 positivity, whereas AMACR showed varied degree of positivity.

In our study, all the 22 cases of Prostatic adenocarcinomas were negative for $\mathrm{p} 63$ and all the 22 cases (100\%) expressed positive immunostaining with AMACR. Among 22 cases of carcinoma prostate there were three cases which were reported as Poorly differentiated adenocarcinoma, which also showed positive expression with AMACR but with less intensity i..e grade 2 intensity. This was due to the process of dedifferentiation.

On reviewing 180 prostate cases, 27 i.e., $15 \%$ atypical cases were delineated. These cases were subjected to further analysis by immunohistochemistry with AMACR and p63 cocktail. In various studies in literature, the incidence of atypical biopsies ranged from $0.4-23 \%$ with a mean $5.5 \%$.

In the index study, based on morphology, clinical details and the interpretation of the IHC with markers p63 and AMACR cocktail, 13 out of 27 cases with a p63 negative staining and moderate to strong positive staining with AMACR were finally categorized as prostatic carcinoma. In conjunction with morphology, a negative AMACR and a positive basal cell layer with p63, 10 cases were labeled as benign and in 4 cases which were initially diagnosed as Atypical small acinar proliferation (suspicious but not diagnostic of malignancy), both AMACR and p63 were negative. In these 4 cases, a diagnosis of atypical small acinar proliferation was retained. Finally, using IHC as an adjuvant, we were able to resolve 23 of the 27 atypical cases $(85.18 \%)$. In this study a diagnosis of adenocarcinoma was made in $48 \%$ of atypical cases.

In Molinie et al. study, 95\% of atypical foci were resolved by using AMACR and p63 cocktail immunostain. ${ }^{[20]}$ In their study a diagnosis of prostatic cancer was made in $40 \%$ of cases which were previously considered as ASAP. They also concluded that p63/P504s cocktail is more specific than CK5/6 alone.

Sanderson et al used AMACR/p63 cocktail to resolve atypical foci. A diagnosis of adenocarcinoma was made in $40 \%$ of cases which were previously diagnosed as atypical. In their study, a final diagnosis was made in $80 \%$ of atypical cases.

The atypical lesions which were diagnosed as benign in our study might be cases of atrophy or reactive atypia or post atrophic hyperplasia, which are common benign mimickers of carcinoma.

When small atypical glands identified by routine H\&E staining are negative for basal cell markers and positive for AMACR/P504S, a malignant diagnosis is established. Although the false-negative rate of P504S staining is very low, it is important to recognize that a negative P504S stain in small "suspicious" glands does not necessarily indicate a benign diagnosis.

Atypical small acinar proliferations which were negative for both AMACR and p63 were either marginally sampled cancer or benign acini with reactive atypia or atrophy or in these cases IHC markers might have failed to work.

According to Epstein et al. study, 34-60\% patients showing atypical small acinar proliferation (ASAP) in the primary biopsies were diagnosed with prostate cancer in repeat biopsy sessions. ${ }^{[21]}$

Hence in these lesions malignancy suspicion is high and these cases were advised for follow-up with digital rectal examination, serum PSA levels, Transrectal ultrasound for every 6months.

Out of 4 cases of ASAP, 2 cases were followed up and 1 case was turned out to be prostatic adenocarcinoma with a Gleason score 6/10 on second biopsy.

Now days, various combination of cocktails are available. In our study we used single colour two antibody cocktail. Triple antibody cocktail with double chromogen are 
available. This antibody cocktail consists of AMACR, p63 and HMWCK.

Recently PIN 4 cocktail antibody was introduced. This antibody is a combination of AMACR, p63, CK5 and CK14. But problem with this PIN4 antibody cocktail is interpretation difficulty. Hence automated analysis sometimes required to interpret a PIN4 slide. ${ }^{[22]}$

\section{Conclusion}

From this study we conclude that AMACR and p63 cocktail antibody is a very useful marker in differentiating benign foci from malignant foci in atypical cases. It helps in diagnosing HGPIN which is a precursor of prostate cancer. False positive and false negative diagnoses are reduced with cocktail antibody. Small lesions in prostatic specimens may disappear in the process of additional specimen cutting for immunohistochemistry with individual antibodies, which can be avoided with this cocktail antibody. Repeat biopsy can be avoided.

\section{References}

1. Garg M, Kaur G, Malhotra V, Garg R. Histopathological spectrum of 364 prostatic specimens including immunohistochemistry with special reference to grey zone lesions. Prostate international. 2013 Dec 1;1(4):146-51.

2. Dabir PD, Ottosen P, Høyer S, Hamilton-Dutoit S. Comparative analysis of three-and two-antibody cocktails to AMACR and basal cell markers for the immunohistochemical diagnosis of prostate carcinoma. Diagnostic pathology. 2012 Dec;7(1):1-6.

3. Consolidated report of population based cancer registries 2001-2004: Incidence and distribution of cancer. Bangalore (IND): Coordinating Unit, National Cancer Registry Programme, Indian Council of Medical Research; 2006.

4. Kumaresan K, Kakkar N, Verma A, Mandal AK, Singh SK, Joshi K. Diagnostic utility of $\alpha$-methylacyl CoA racemase (P504S) \& HMWCK in morphologically difficult prostate cancer. Diagnostic Pathology. 2010 Dec 1;5(1):83.

5. Shah RB, Zhou M, LeBlanc M, Snyder M, Rubin MA. Comparison of the basal cell-specific markers, $34 \beta E 12$ and p63, in the diagnosis of prostate cancer. Am J Surg Pathol. 2002 Sep 1;26(9):1161-8.

6. Signoretti S, Waltregny D, Dilks J, Isaac B, Lin D, Garraway L, Yang A, Montironi R, McKeon F, Loda M. p63 is a prostate basal cell marker and is required for prostate development. Am J Pathol. 2000 Dec 1;157(6):1769-75.

7. Parsons JK, Gage WR, Nelson WG, De Marzo AM. p63 protein expression is rare in prostate adenocarcinoma: implications for cancer diagnosis and carcinogenesis. Urology. 2001 Oct 1;58(4):619-24.

8. Beach R, Gown AM, De Peralta-Venturina MN, Folpe AL, Yaziji H, Salles PG, Grignon DJ, Fanger GR, Amin MB.
P504S immunohistochemical detection in 405 prostatic specimens including 376 18-gauge needle biopsies. Am J Surg Pathol. 2002 Dec 1;26(12):1588-96.

9. Shah RB, Tadros Y, Brummell B, Zhou M. The diagnostic use of ERG in resolving an "atypical glands suspicious for cancer" diagnosis in prostate biopsies beyond that provided by basal cell and $\alpha$-methylacyl-CoA-racemase markers. Human pathology. 2013 May 1;44(5):786-94.

10. Kuroda N. Application of combined immunohistochemical panel of AMACR (P504S)/p63 cocktail, cytokeratin 5 and D2-40 to atypical glands in prostatic needle biopsy. The Malaysian journal of pathology. 2014 Dec 1;36(3):169.

11. $\mathrm{Xu}$ J, Stolk JA, Zhang $\mathrm{X}$, Silva SJ, Houghton RL, Matsumura M, Vedvick TS, Leslie KB, Badaro R, Reed SG. Identification of differentially expressed genes in human prostate cancer using subtraction and microarray. Cancer Res. 2000 Mar 15;60(6):1677-82.

12. Luo J, Zha S, Gage WR, Dunn TA, Hicks JL, Bennett CJ, Ewing CM, Platz EA, Ferdinandusse S, Wanders RJ, Trent JM. $\alpha$-Methylacyl-CoA racemase: a new molecular marker for prostate cancer. Cancer Res. 2002 Apr 15;62(8):2220-6.

13. George E, Thomas S. A histopathologic survey of prostate disease in the sultanate of oman. Internet J Pathol 2005;3(2).

14. Barakzai MA, Mubarak M, Kazi JI. Histopathological lesions in transrectal ultrasound guided biopsies of prostate in patients with raised serum prostate specific antigen: a preliminary report. Nephro-Urol Mon 2011;3:186-90.

15. Anjorin AS, Adeniji KA, Ogunsulire IA. Histopathological study of prostatic lesions in Ilorin, Nigeria. Cent Afr J Med 1998;44:72-5.

16. Evans AJ. $\alpha$-Methylacyl CoA racemase (P504S): overview and potential uses in diagnostic pathology as applied to prostate needle biopsies. Journal of clinical pathology. 2003 Dec 1;56(12):892-7.

17. Leav I, McNeal JE, Ho SM, Jiang Z. $\alpha$-Methylacyl-CoA racemase (P504S) expression in evolving carcinomas within benign prostatic hyperplasia and in cancers of the transition zone. Human pathology. 2003;34(3):228-33.

18. Ananthanarayanan V, Deaton RJ, Yang XJ, Pins MR, Gann PH. Alpha $\square$ methylacyl $\square$ CoA racemase (AMACR) expression in normal prostatic glands and high $\square$ grade prostatic intraepithelial neoplasia (HGPIN): Association with diagnosis of prostate cancer. The Prostate. 2005 Jun $1 ; 63(4): 341-6$.

19. Sanderson SO, Sebo TJ, Murphy LM, Neumann R, Slezak J, Cheville JC. An analysis of the p63/ $\alpha$-methylacyl coenzyme A racemase immunohistochemical cocktail stain in prostate needle biopsy specimens and tissue microarrays. American journal of clinical pathology. 2004 Feb 1;121(2):220-5.

20. Molinié V, Fromont G, Sibony M, Vieillefond A, Vassiliu V, Cochand-Priollet B, Hervé JM, Lebret T, Baglin AC. Diagnostic utility of a p63/ $\alpha$-methyl-CoA-racemase (p504s) 
cocktail in atypical foci in the prostate. Modern Pathology. 2004 Oct;17(10):1180-90.

21. Epstein JI, Herawi M. Prostate needle biopsies containing prostatic intraepithelial neoplasia or atypical foci suspicious for carcinoma: implications for patient care. The Journal of urology. 2006 Mar;175(3):820-34.
22. Sabata B, Babenko B, Monroe R, Srinivas C. Automated analysis of pin-4 stained prostate needle biopsies. InInternational Workshop on Prostate Cancer Imaging 2010 Sep 24 (pp. 89-100). Springer, Berlin, Heidelberg.

*Corresponding author:

Sunandalakshmi GV, Assistant Professor, Department of pathology, Narayana Medical College, Nellore, Andhra Pradesh

Email: sunandasankar19@gmail.com

Date of Submission : 25/01/2020

Date of Acceptance : 05/10/2020

Financial or other Competing Interests: None.

Date of Publication : 30/11/2020 